\title{
April 27 Highlights
}

\section{Contrast-enhanced MR angiography for carotid stenosis}

In a prospective study, U-King-Im et al. evaluated noninvasive carotid imaging in 167 patients with suspected carotid stenosis. Contrast-enhanced MRA compared favorably with conventional intra-arterial DSA. They considered that clinical decisions would potentially be altered in only $6 \%$ of cases.

see page 1282

The accompanying editorial by William Powers notes that contrast enhanced MR angiography (CEMRA) is the latest noninvasive technique developed to provide images of the carotid arteries. Because even in the best of hands, DSA carries a risk of 1 to $4 \%$ for stroke and death, Powers considers why DSA is still considered the gold standard: he points out that all the randomized, controlled, clinical trials that demonstrated the superiority of endarterectomy over medical therapy for the prevention of subsequent strokes in symptomatic patients employed intra-arterial catheter contrast arteriography to determine the degree of stenosis. Thus, the data regarding the benefits of surgery only apply when the degree of stenosis is determined using the same technique. U-King-Im et al. conclude that their data on CEMRA support its safe use instead of DSA. Powers reviews reasons why reports on newer technologies often may be overly optimistic and points out that the U-King-Im et al. comparison of CEMRA with DSA shows that CEMRA generally overestimated the degree of arteriographic stenosis and did not correlate closely enough to provide data necessary to accurately determine the benefit of endarterectomy in individual patients.

see page 1246

\section{Use of the new AED in newly diagnosed epilepsy}

In this practice parameter, French et al. assess several new AED-gabapentin, lamotrigine, topiramate, tiagabine, oxcarbazepine, levetiracetam, and zonisamidefor which effectiveness has been established in patients with newly diagnosed epilepsy, and identify which subpopulations have been studied. They also evaluate adverse events and safety, and present the available data comparing older and newer AED.

see page 1252

\section{Use of the new AED in refractory epilepsy}

A second parameter by French et al. evaluates studies performed in patients with refractory epilepsy with the same seven AED. Studies in adjunctive and monotherapy use are outlined separately. Evidence for efficacy of each new AED in partial and generalized epilepsy in both adults and children is presented.

see page 1261

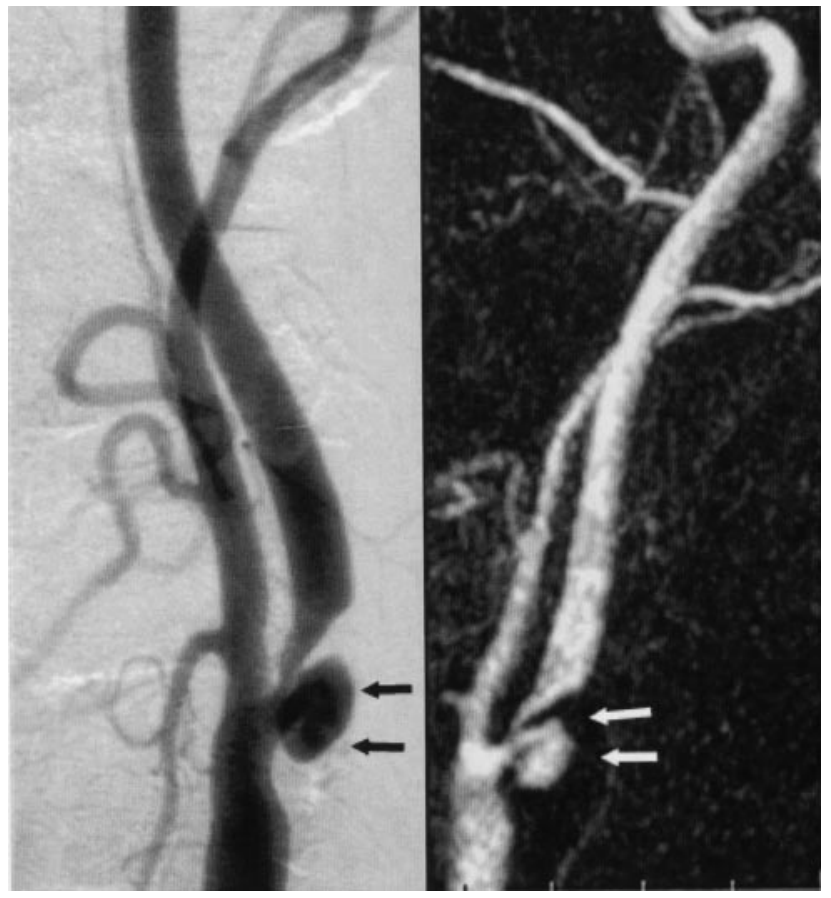

DSA (left) and CEMRA (right) depicting a severe stenosis of the internal carotid artery with a large ulcer crater (arrows) into the atheromatous plaque.

\section{Trans-vascular delivery of genes to the brain}

Schlachetzki et al. found that potentially therapeutic genes can now be delivered to all parts of the brain with simple IV administration without the use of viruses. Genes are encapsulated in liposomes, which are targeted across the blood-brain barrier with genetically engineered molecular Trojan horses.

see page 1275

\section{Long-term sequelae in children after cerebellar astrocytoma surgery}

Aarsen et al. studied long-term effects on neurologic, neuropsychological, and behavioral functioning in 23 children treated surgically for a cerebellar pilocytic astrocytoma. All children showed at least some deficits, including apraxia, neglect, dysarthria, and language, attention, visual-spatial, executive, memory, and behavioral problems.

see page 1311 


\section{Mutations in myotilin cause myofibrillar myopathy (MFM)}

Selcen and Engel found that mutations in myotilin, a Z-disk related protein, cause myofibrillar myopathy. The patients had distal weakness, peripheral neuropathy, and cardiomyopathy. Myotilin mutations were previously detected in two kinships with LGMD1A. The present finding modifies the concept of LGMD1A and should spur the search for mutations in Z-disk related proteins in MFM patients.

see page 1363

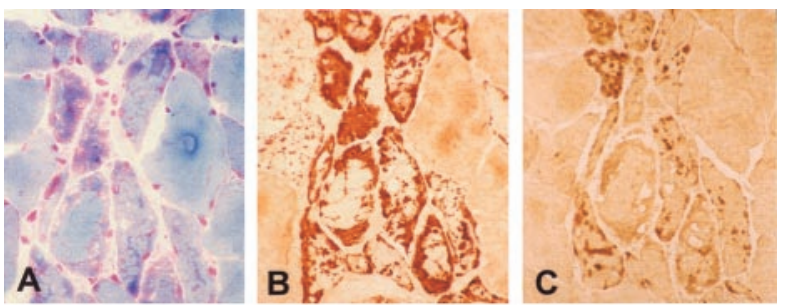

(A) Trichrome; (B) myotilin; (C) ubiquitin.

In the accompanying editorial Karpati and Sinnreich note that these observations in six MFM patients expand the genetic heterogeneity of MFM; provide additional molecular knowledge about the biology of the $Z$ disc and myofibrillar organization; provide another example of the important role of putative modifying genes in inconsistent genotypephenotype relationships; and illustrate how clever scrutiny of morphologic data provides the clue for discovering diseasecausing genes.

see page 1248

\section{Chronic daily headache with analgesic overuse}

Colás et al. interviewed 4,855 unselected subjects. The prevalence of $\mathrm{CDH}$ with analgesic overuse was $1.4 \%$ (2.6\% in women). Transformed migraine (prevalence $0.9 \%)$ was more frequent than chronic tension-type headache (0.4\%). CDH subjects showed a significant decrease in SF-36 health-related scores as compared with controls, emphasizing the public health impact of $\mathrm{CDH}$ with analgesic overuse.

see page 1338

\section{Controlled trial of pergolide for restless legs syndrome}

Trenkwalder et al. studied the efficacy of pergolide in 100 patients with idiopathic RLS. Patients were monitored with polysomnography and recently validated scales at multiple time points. In the 6 -week randomized, controlled trial, pergolide substantially improved both polysomnographic and subjective sleep measures. In open trial it maintained its efficacy for a year.

see page 1391

\section{Reversible parkinsonism in patients exposed to sodium valproate}

Easterford et al. examined 50 consecutive patients taking sodium valproate monotherapy identifying three cases with unequivocal parkinsonism and raised UPRDS scores. Cessation of therapy was associated with resolution of parkinsonian features. B-CIT SPECT in these patients was negative, arguing against unmasking of concomitant Parkinson's disease.

see page 1435

\section{No RT-PCR detection of enterovirus RNA in ALS specimens}

Nix et al. attempted to reproduce the reported detection of enterovirus RNA in specimens from ALS patients. No enterovirus RNA could be detected in freshfrozen blinded specimens, tested in parallel at two independent laboratories, using a sensitive semi-nested RT-PCR assay, internal specimen control targets, and sequencing for amplicon confirmation.

see page 1372

The accompanying editorial by Jubelt and Lipton notes that the weight of evidence, including Nix et al., is now against an enteroviral cause of ALS. However, the data are not exclusionary and questions remain. First, the techniques used may make a difference, e.g., frozen vs fixed tissue, single amplification vs nested or semi-nested amplification. Second, many different primers have been used in these studies. These various primers probably do not have similar specificities for both polioviruses and non-polio enteroviruses. Third, the sensitivity of the various RT-PCR techniques used is not often clearly stated. The use of a limiting dilution analysis of known quantities of virus in spiked samples would be useful for determining the lower limits of detection. Fourth, the state of diseased tissue may be of importance. In the various studies, most tissues were probably obtained late in the disease, many months after anterior horn cells had died. Thus, it would be worthwhile to know if any of the spinal cord samples corresponded to strong muscles actively deteriorating at the time of death (early diseased), at a time when a virus might be more likely to be detected.

see page 1250 


\section{Neurology}

April 27 Highlights

Neurology 2004;62;1244-1245

DOI 10.1212/WNL.62.8.1244

\section{This information is current as of April 26, 2004}

\section{Updated Information \&}

Services

Permissions \& Licensing

Reprints including high resolution figures, can be found at: http://n.neurology.org/content/62/8/1244.full

Information about reproducing this article in parts (figures,tables) or in its entirety can be found online at:

http://www.neurology.org/about/about_the_journal\#permissions

Information about ordering reprints can be found online:

http://n.neurology.org/subscribers/advertise

Neurology ${ }^{\circledR}$ is the official journal of the American Academy of Neurology. Published continuously since 1951, it is now a weekly with 48 issues per year. Copyright. All rights reserved. Print ISSN: 0028-3878. Online ISSN: 1526-632X.

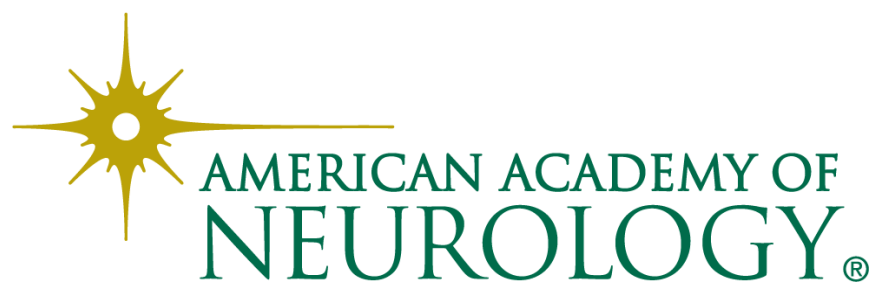

